\title{
Fatty acid composition of serum lipid classes in mice following allergic sensitisation with or without dietary docosahexaenoic acid-enriched fish oil substitution
}

\author{
Ralph Rühl ${ }^{1}{ }^{*}$, Christin Koch $^{2,3}$, Tamás Marosvölgyi ${ }^{4}$, Johanna Mihály ${ }^{1}$, Florian J. Schweigert ${ }^{3}$, \\ Margitta Worm ${ }^{2}$ and Tamás Decsi ${ }^{4}$ \\ ${ }^{1}$ Department of Biochemistry and Molecular Biology, Medical and Health Science Centre, University of Debrecen, \\ Nagyerdei Krt. 98, H-4012 Debrecen, Hungary \\ ${ }^{2}$ Department of Dermatology and Allergy, Charité-Universitätsmedizin, Berlin, Germany \\ ${ }^{3}$ Department of Nutritional Physiology and Pathophysiology, Institute of Nutritional Science, University of Potsdam, \\ Potsdam-Rehbrücke, Germany \\ ${ }^{4}$ Department of Paediatrics, University of Pécs, Pécs, Hungary \\ (Received 19 June 2007 - Revised 1 October 2007 - Accepted 4 October 2007 - First published online 16 November 2007)
}

Dietary fatty acids have been shown to influence allergic sensitisation. Both $n-3$ and $n-6$ PUFA are involved in targeted mediation of inflammatory responses during allergic sensitisation and manifestation of atopic diseases. In the present experiments we investigated whether supplementation of DHA-enriched fish oil partly substituting dietary sunflower-seed oil, in comparison with sunflower-seed oil, supplemented to mice influences fatty acid composition of serum lipid classes. The effects of the two different diets were also investigated depending on allergic sensitisation. Supplementation of DHA and EPA in doses of 2 and $0.12 \%(\mathrm{w} / \mathrm{w})$ to non-sensitised and sensitised mice resulted in significantly increased percentile contributions of DHA to all lipid classes. In contrast, serum values of the $n$-6 PUFA arachidonic acid (AA) were significantly lower, both in nonsensitised and sensitised mice fed the DHA-enriched diet. The fatty acid composition of serum lipids also reflected allergic sensitisation: the EPA:AA ratio in TAG, cholesteryl esters and phospholipids in non-supplemented animals fell to 23, 29 and $29 \%$ respectively of the original value after allergic sensitisation, whereas it decreased to 70,80 and $76 \%$ respectively only in the animals supplemented with DHA. In summary, allergic sensitisation alone decreased significantly the EPA:AA ratios in serum TAG, while concomitant supplementation of DHA-enriched fish oil ameliorated this decrease. We postulate from the present results that the amelioration of the severity of allergic sensitisation after DHA supplementation may be linked to altered ratios of the eicosanoid precursors EPA and AA as well as DHA needed for further metabolic activation to pro- or anti-inflammatory bioactive lipids.

Docosahexaenoic acid: Arachidonic acid: Eicosapentaenoic acid: Dihomo- $\gamma$-linolenic acid: Allergic sensitisation: $n$-3 Polyunsaturated fatty acids

Fatty acids in the mammalian organism are mainly present in esterified form, and in lower concentrations as NEFA. Phospholipid- and cholesteryl esters are the most abundant forms of fatty acid esters in membranes and they occur in addition to TAG also in the serum. PUFA are important nutrients in the human $\operatorname{diet}^{(1-3)}$. Various plant oils, such as sunflowerseed and soyabean oil, as well as meat products, contain mainly $n$-6 PUFA with linoleic acid, $\gamma$-linolenic acid and arachidonic acid (AA) as major constituents, whereas fish and linseed oil contain mainly the $n$-3 PUFA: $\alpha$-linolenic acid in linseed oil and EPA and DHA in marine oils such as fish oil.

Three PUFA (EPA from the $n-3$ series and AA as well as dihomo- $\gamma$-linolenic acid (DHGLA) from the $n$ - 6 series) are substrates for various lipo-oxygenases (LOX) and cyclo-oxy- genases (COX). They can be further metabolised to highly bioactive eicosanoids such as leucotrienes and PG (Miles et $a l^{(4)}$; for a review, see Rustan \& Drevon $\left.{ }^{(3)}\right)$, as well as lipoxins, neuroprotectins and resolvins which mainly originate from DHA and EPA (for reviews, see Serhan ${ }^{(5)}$ and Serhan \& Savill $\left.^{(6)}\right)$. Although DHA is not directly involved in eicosanoid synthesis, retroconversion of DHA to EPA has been reported $^{(7)}$

Increased dietary supplementation with plant oils rich in $n-6$ PUFA has been associated with increased production of proinflammatory mediators ${ }^{(8,9)}$, whereas dietary supplementation with oils rich in the $n-3$ PUFA EPA and DHA induced antiinflammatory effects in in vitro as well as in vivo experiments $^{(2,8-10)}$. Moreover, a higher dietary intake of $n-3$

Abbreviations: AA, arachidonic acid; COX, cyclo-oxygenase; CTRL, control (basal diet); DHGLA, dihomo- $\gamma$-linolenic acid; LOX, lipo-oxygenase; OVA, ovalbumin.

* Corresponding author: Dr Ralph Rühl, fax +36 52314 989, email rruehl@dote.hu 
PUFA was associated with lower incidences of allergic sensitisation and atopic diseases in several studies ${ }^{(11-13)}$. Supplementation with various forms of oils rich in DHA or fish oil ameliorated symptoms of allergic sensitisation in atopic children ${ }^{(14)}$, mixed population of atopic children and adults $^{(15}$, atopic dogs ${ }^{16-18)}$ and in mouse allergy models ${ }^{(19,20)}$.

Allergic sensitisation is reflected by an altered T-cell phenotype and cytokine responses (for reviews, see Worm \& $\mathrm{Henz}^{(21)}$ and Bacharier \& Geha $\left.{ }^{(22)}\right)$. In particular, PG of the $\mathrm{PgE}$ class and leucotrienes of the 4-series were associated with a shift from T-helper cell 1 to T-helper cell 2 response $(\mathrm{Th} 1 \rightarrow \mathrm{Th} 2 \text { shift })^{(4,23,24)}$. Leucotrienes originating from $n-3$ PUFA are, in general, less potent pro-inflammatory substances than their analogues originating from $n-6 \mathrm{PUFA}^{(25)}$, while PG originating from different fatty acids are almost equipotent ${ }^{(4)}$.

The aim of the study was to find out how allergic sensitisation, DHA-enriched fish oil supplementation and allergic sensitisation with concomitantly DHA-enriched fish oil supplementation influence the serum percentile distribution of cholesteryl-, TAG- and phospholipid esters of the fatty acids and various ratios of eicosanoid precursor PUFA such as EPA, DHA and AA.

\section{Materials and methods}

\section{Animal experiments}

The animal experiments were performed in the facilities of the Max Rubner Laboratory of the German Institute of Human Nutrition (DIfE) (Potsdam-Rehbrücke, Germany). The respective ethical authorities from the Land Brandenburg approved the experiment. A second set of experiments, for investigation of the influence of $\mathrm{Al}(\mathrm{OH})_{3}$ on lipid distribution, was performed in the Laboratory Animal Core Facility of the University of Debrecen (Debrecen, Hungary). The experiments were performed according to the ethical guidelines of the Republic of Hungary.

All mice were female Balb/c adult (age 10-12 weeks) mice (Mus musculus) and were obtained from Charles River (Sulzfeld, Germany). The animals were kept under controlled conditions at room temperature $\left(21 \pm 1^{\circ} \mathrm{C}\right)$ and constant relative humidity $(55 \pm 5 \%)$ and a $12 \mathrm{~h}$ light $-12 \mathrm{~h}$ dark cycle with light between 06.00 and 18.00 hours

Food and water were administered ad libitum. The composition of the basal diet was $20 \%$ casein, $8 \%$ sucrose, $50 \%$ wheat starch, $10 \%$ sunflower-seed oil, $5 \%$ cellulose, $5 \%$ mineral mix (Mineral-Spurenelemente-Vormischung C1000; Altromin, Lage, Germany) and $2 \%$ vitamin mix (VitaminVormischung C1000; Altromin) ${ }^{(26)}$. In the diet supplemented with DHA-enriched fish oil, $40 \%$ of the sunflower-seed oil was substituted by DHA-enriched fish oil (DHA 500TG SR from tuna; Croda $\mathrm{GmbH}$, Nettetal, Germany) yielding a mass content of $2 \%$ DHA and $0.12 \%$ EPA in the animals' diet. The composition of the two diets is displayed in Table 1. The group fed the basal diet and the group fed the DHA-supplemented diet each contained twelve mice; for the second experiment using aluminium hydroxide $\left(\mathrm{Al}(\mathrm{OH})_{3}\right)$ treatment for the group fed the basal diet and the DHA-supplemented group, six mice per group were used.

Before supplementation with the basal and DHA-enriched fish oil diet the mice were fed with a standard mouse diet
Table 1. Percentage fatty acid composition of the administered food ${ }^{\star}$

\begin{tabular}{lcc}
\hline Fatty acid (\%) & Control diet & $\begin{array}{c}\text { Diet supplemented } \\
\text { with DHA-enriched fish oil }\end{array}$ \\
\hline Sum of SFA & 12.4 & 8.3 \\
Sum of MUFA & 22.7 & 17.6 \\
Sum of $n$-6 PUFA & 64.3 & 42.7 \\
Linoleic acid & 64.0 & 40.9 \\
Arachidonic acid & 0.01 & 0.56 \\
Sum of $n$-3 PUFA & 0.63 & 31.4 \\
EPA & 0.12 & 3.6 \\
DHA & 0.26 & 23.4 \\
\hline
\end{tabular}

${ }^{*}$ Data are percentages $(\mathrm{w} / \mathrm{w})$ of dry animal food.

from Sniff (Soest, Germany) and for the second set of experiments using $\mathrm{Al}(\mathrm{OH})_{3}$ treatment the mice were fed with a standard mouse diet from Altromin (Lage, Germany). At 1 week after the start of the supplementation with the basal and the DHA-enriched fish oil diet, six mice per group were sensitised with ovalbumin (OVA) and six mice were treated with PBS. In the second set of experiments just $\mathrm{Al}(\mathrm{OH})_{3}$ treatment was performed in a comparable manner. Each group was treated either with three intraperitoneal injections of OVA or PBS (or $\mathrm{Al}(\mathrm{OH})_{3}$ in PBS) after a time interval of 1 week. OVA injections were made according to the protocol previously described $^{(27)}$; briefly, $10 \mu \mathrm{g}$ OVA absorbed to $1.5 \mathrm{mg}$ $\mathrm{Al}(\mathrm{OH})_{3}$ in $100 \mu \mathrm{l} \mathrm{PBS}$ was given to each animal. The mice were killed by decapitation 4 weeks after the dietary and 3 weeks after the OVA or PBS treatments. At 20-30 min after the collection the blood was centrifuged at $1300 \mathrm{~g}$ for $3 \mathrm{~min}$ and serum was obtained. Serum was stored at $-80^{\circ} \mathrm{C}$ until analysis.

\section{Fatty acid analysis}

For the analysis of plasma fatty acid profiles, frozen plasma samples were thawed, and then the three internal standards (dipentadecanoylphosphatidylcholine, cholesteryl pentadecanoate and cholesteryl tripentadecanoate) were added. Lipids were extracted by the addition of $3 \mathrm{ml}$ chloroform and $1 \mathrm{ml}$ methanol according to the method of Folch et al. ${ }^{(28)}$. The mixture was vortexed at $2000 \mathrm{rpm}$ for $10 \mathrm{~min}$, and then the lower layer was aspirated into vials and evaporated under an $\mathrm{N}_{2}$ stream. Lipid extracts were reconstituted in $70 \mu \mathrm{l}$ chloroform and lipid classes were separated by TLC. The solvents for TLC of plasma lipids were as follows: hexane-diethyl etherchloroform-acetic acid (21:6:3:1, by vol.) followed by chloroform-methanol-water $(65: 25: 4$, by vol.). The bands were stained with dichlorofluorescein, removed by scraping and transesterified in $3 \mathrm{M}-\mathrm{HCl}-$ methanol solution at $84^{\circ} \mathrm{C}$ for $45 \mathrm{~min}$.

Fatty acids were analysed by high-resolution capillary GC using a Finnigan 9001 gas chromatograph (Finnigan/Tremetrics Inc., Austin, TX, USA) with split injection (ratio $1: 25$ ), automatic sampler (A200SE; CTC Analytic, Zwingen, Switzerland) and flame ionisation detector with a DB-23 cyanopropyl column of $40 \mathrm{~m}$ length (J \& W Scientific, Folsom, CA, USA). The temperature program was the following: temperature of injector at $80^{\circ} \mathrm{C}$ for $0.1 \mathrm{~min}$, temperature increase by $180^{\circ} \mathrm{C} / \mathrm{min}$ up to $280^{\circ} \mathrm{C}$, temperature of detector 
at $280^{\circ} \mathrm{C}$, temperature of column area at $60^{\circ} \mathrm{C}$ for $0.2 \mathrm{~min}$, temperature increase by $40^{\circ} \mathrm{C} / \mathrm{min}$ up to $180^{\circ} \mathrm{C}, 5 \mathrm{~min}$ isothermal period, temperature increase by $1.5^{\circ} \mathrm{C} / \mathrm{min}$ up to $200^{\circ} \mathrm{C}$, $8.5 \mathrm{~min}$ isothermal period, temperature increase by $40^{\circ} \mathrm{C} / \mathrm{min}$ up to $240^{\circ} \mathrm{C}$ and $13 \mathrm{~min}$ isothermal period. The constant linear velocity was $0.3 \mathrm{~m} / \mathrm{s}$ (referred to $100^{\circ} \mathrm{C}$ ). Peak identification was verified by comparison with authentic standards. Fatty acid results were expressed as percentages (w/w) of fatty acids detected with a chain length between twelve and twenty-four carbon atoms.

\section{Enzyme-linked immunosorbent assay analysis}

Plates were coated overnight with anti-mouse EM95.3 (5 $\mu \mathrm{g} /$ $\mathrm{ml}$; all monoclonal antibodies used were kindly provided by Dr Lamer, MPI, Freiburg, Germany) diluted in $0 \cdot 1 \mathrm{M}$-bicarbonate buffer. After blocking with $3 \%$ milk powder-PBS, sera (diluted in $1 \%$ milk powder-PBS) were incubated overnight and were detected with biotin-conjugated anti-mouse 84 1-C $(1 \mu \mathrm{g} / \mathrm{ml})$. The reaction was developed with streptavidin peroxidase and tetramethylbenzidine (both from Sigma, Dreieich, Germany) and was stopped with $1 \mathrm{M}$-sulfuric acid. The plates were measured at $450 / 490 \mathrm{~nm}$ and the amount of total IgE was calculated according to the standard curve. The validity was assessed by using a standard with known concentrations for total IgE and by determination of $50 \%$ saturation for OVA-specific IgE ELISA. Furthermore, all sera were measured in serial dilutions.

\section{Statistics}

Standard deviations were calculated with SPSS version 15.0 (SPSS Inc., Chicago, IL, USA) software for Windows using a two-factor independent Mann-Whitney test; a value of $P<0.05$ was used to determine statistical significance.

\section{Results}

Here we discuss separately the effects of diet (basal diet (control; CTRL) and PBS injections (CTRL-PBS) v. DHAenriched fish oil supplementation and PBS injections (DHA-PBS); basal diet and OVA injections (CTRL-OVA) $v$. DHA-enriched fish oil supplementation and OVA injections (DHA-OVA)) and the effect of allergic sensitisation without dietary supplementation (basal diet with PBS injections (CTRL-PBS) $v$. basal diet with OVA injections (CTRLOVA)) and with dietary DHA supplementation (DHAenriched fish oil supplementation with PBS injections (DHA-PBS) $v$. DHA-enriched fish oil supplementation with OVA injections (DHA-OVA)). In a second set of experiments we performed $\mathrm{Al}(\mathrm{OH})_{3}$ treatments to the animals fed the basal diet and the animals supplemented with the DHA-enriched fish oil. Fatty acid composition of plasma cholesteryl esters, phospholipids and TAG are shown in Tables 2, 3, 4 and 6, respectively. Ratios of selected $n-3$ and $n-6$ PUFA such as EPA, DHA and DHGLA TAG are shown in Tables 5 and 6 .

\section{Effect of dietary docosahexaenoic acid-enriched fish oil supplementation without allergic sensitisation (control diet and phosphate-buffered saline treatment $\mathrm{v}$. docosahexaenoic acid-enriched diet and phosphate-buffered saline treatment)}

The sum of SFA and MUFA were comparable between both groups, except for higher values of MUFA in TAG in the control animals. $n$-3 PUFA levels were strongly increased after DHA-enriched fish oil supplementation in all the three lipid classes. Inconsistent results were seen in the individual

Table 2. Fatty acid composition of plasma cholesteryl ester lipids in mice fed either a diet supplemented with docosahexaenoic acid-enriched fish oil or a control diet (CTRL) and subsequently treated either with ovalbumin (OVA) or phosphate-buffered salineł

(Mean values and standard deviations)

\begin{tabular}{|c|c|c|c|c|c|c|c|c|}
\hline \multirow{2}{*}{$\begin{array}{l}\text { Treatment... } \\
\text { Fatty acid }\end{array}$} & \multicolumn{2}{|c|}{ CTRL-PBS } & \multicolumn{2}{|c|}{ DHA-PBS } & \multicolumn{2}{|c|}{ CTRL-OVA } & \multicolumn{2}{|c|}{ DHA-OVA } \\
\hline & Mean & SD & Mean & SD & Mean & SD & Mean & SD \\
\hline Sum of SFA & 8.96 & 0.34 & 11.47 & 0.63 & 6.09† & 0.18 & $12 \cdot 78^{*}$ & 0.68 \\
\hline Sum of MUFA & $12 \cdot 00$ & 0.51 & $11 \cdot 36$ & 0.44 & $10 \cdot 97$ & 0.31 & $13 \cdot 24$ & 0.27 \\
\hline $18: 2 n-6(\mathrm{LA})$ & 31.53 & $15 \cdot 44$ & $43 \cdot 07^{\star}$ & $4 \cdot 10$ & $32 \cdot 73$ & $9 \cdot 22$ & $43.02^{*}$ & 2.07 \\
\hline $18: 3 n-6$ (GLA) & 0.51 & 0.32 & $0 \cdot 29^{\star}$ & 0.06 & 0.42 & 0.04 & 0.55 & 0.73 \\
\hline $20: 2 n-6$ & 0.30 & 0.17 & $0.16^{\star}$ & 0.19 & 0.13 & 0.02 & 0.19 & 0.17 \\
\hline $20: 3 n-6$ (DHGLA) & 0.58 & 0.17 & $0.95^{*}$ & 0.10 & 0.48 & 0.06 & $1 \cdot 13^{*}$ & 0.25 \\
\hline $20: 4 n-6$ (AA) & $42 \cdot 51$ & $12 \cdot 14$ & $20 \cdot 16^{\star}$ & 5.00 & $46 \cdot 61$ & $7 \cdot 86$ & $18 \cdot 84^{*}$ & 5.49 \\
\hline $22: 4 n-6$ & 0.63 & 0.25 & $0.15^{\star}$ & 0.06 & 0.54 & 0.15 & $0.14^{\star}$ & 0.04 \\
\hline $22: 5 n-6$ & 0.91 & 0.53 & 0.18 & 0.12 & 0.08 & 0.03 & 0.58 & 0.69 \\
\hline Sum of $n-6$ PUFA & 76.98 & 0.77 & $64 \cdot 96^{*}$ & 1.02 & 80.99 & 0.42 & $64 \cdot 44^{*}$ & 0.65 \\
\hline $18: 3 n-3(\mathrm{ALA})$ & 0.05 & 0.01 & $0 \cdot 24$ & 0.39 & 0.03 & 0.03 & $0 \cdot 19^{\star}$ & 0.27 \\
\hline $20: 3 n-3$ & 0.29 & 0.26 & $1.04^{*}$ & 1.35 & $0.12 \dagger$ & 0.06 & $0.53^{\star}$ & 0.17 \\
\hline $20: 5 n-3$ (EPA) & 0.05 & 0.05 & $4 \cdot 21^{*}$ & 1.53 & 0.02 & 0.01 & $3 \cdot 12^{*}$ & $1 \cdot 13$ \\
\hline $22: 5 n-3$ (DPA) & 0.03 & 0.01 & $0.15^{\star}$ & 0.02 & 0.03 & 0.01 & 0.15 & 0.08 \\
\hline $22: 6 n-3$ (DHA) & 1.64 & 0.75 & $6 \cdot 58^{*}$ & $2 \cdot 84$ & 1.75 & 0.34 & $5 \cdot 55^{\star}$ & $2 \cdot 61$ \\
\hline Sum of $n-3$ PUFA & 2.07 & 0.15 & $12 \cdot 20^{*}$ & 0.63 & 1.95 & 0.08 & $9 \cdot 54^{*}$ & 0.67 \\
\hline
\end{tabular}

LA, linoleic acid; GLA, $\gamma$-linolenic acid; DHGLA, dihomo- $\gamma$-linolenic acid; AA, arachidonic acid; ALA, $\alpha$-linolenic acid; DPA, docosapentaenoic acid.

* Mean value is significantly different from that of the CTRL animals $(P<0.05)$

† Mean value is significantly different from that of the PBS-treated animals $(P<0.05)$.

$\ddagger$ Data are percentages $(\mathrm{w} / \mathrm{w})$ calculated on the basis of six independent samples. 
Table 3. Fatty acid composition of plasma phospholipids in mice fed either with a diet supplemented with docosahexaenoic acid-enriched fish oil or a control diet (CTRL) and subsequently treated either with ovalbumin (OVA) or phosphate-buffered saline‡

(Mean values and standard deviations)

\begin{tabular}{|c|c|c|c|c|c|c|c|c|}
\hline \multirow{2}{*}{$\begin{array}{l}\text { Treatment... } \\
\text { Fatty acid }\end{array}$} & \multicolumn{2}{|c|}{ CTRL-PBS } & \multicolumn{2}{|c|}{ DHA-PBS } & \multicolumn{2}{|c|}{ CTRL-OVA } & \multicolumn{2}{|c|}{ DHA-OVA } \\
\hline & Mean & SD & Mean & SD & Mean & SD & Mean & SD \\
\hline Sum of SFA & $49 \cdot 01$ & 0.72 & 45.97 & 0.51 & $46 \cdot 06$ & 0.29 & $52 \cdot 99^{*} \dagger$ & 0.44 \\
\hline Sum of MUFA & $9 \cdot 13$ & 0.32 & $10 \cdot 01$ & 0.33 & $10 \cdot 08$ & 0.27 & 11.70 & 0.32 \\
\hline $18: 2 n-6(\mathrm{LA})$ & $22 \cdot 75$ & 1.58 & 24.72 & 2.85 & $22 \cdot 11$ & 1.98 & $20.82 \dagger$ & 1.23 \\
\hline $18: 3 n-6$ (GLA) & 0.10 & 0.04 & 0.67 & 0.59 & 0.14 & 0.12 & $0.05^{\star}$ & 0.02 \\
\hline $20: 2 n-6$ & 0.31 & 0.04 & 0.27 & 0.04 & 0.33 & 0.04 & 0.30 & 0.03 \\
\hline $20: 3 n-6$ (DHGLA) & 1.00 & 0.19 & $1.63^{\star}$ & 0.30 & 1.03 & 0.12 & $1 \cdot 64^{*}$ & 0.32 \\
\hline $20: 4 n-6$ (AA) & 14.01 & 3.63 & $6 \cdot 10^{*}$ & $1 \cdot 78$ & $15 \cdot 88$ & 1.98 & $5 \cdot 48^{\star}$ & 1.46 \\
\hline $22: 4 n-6$ & 1.50 & 0.70 & $0.44^{\star}$ & 0.25 & 1.69 & 0.56 & $0.25^{\star}$ & 0.07 \\
\hline $22: 5 n-6$ & 0.03 & 0.01 & $0.49^{*}$ & 0.81 & 0.09 & $0 \cdot 10$ & $0 \cdot 13$ & 0.15 \\
\hline Sum of $n-6$ PUFA & 39.69 & 0.70 & $34 \cdot 32$ & 0.82 & $41 \cdot 26$ & 0.51 & $28 \cdot 68^{*} \dagger$ & 0.34 \\
\hline 18:3n-3 (ALA) & 0.02 & 0.01 & 0.24 & 0.22 & 0.04 & 0.03 & 0.05 & 0.03 \\
\hline $20: 3 n-3$ & 0.12 & 0.08 & 0.40 & 0.22 & 0.13 & 0.08 & $0 \cdot 18^{\star}$ & 0.12 \\
\hline $20: 5 n-3$ (EPA) & 0.007 & 0.005 & $0.94^{*}$ & 0.32 & $0.003 \dagger$ & 0.001 & $0.64^{\star}$ & 0.17 \\
\hline $22: 5 n-3$ (DPA) & 0.02 & 0.01 & $0.27^{\star}$ & 0.08 & 0.03 & 0.00 & $0.23^{\star}$ & 0.04 \\
\hline $22: 6 n-3$ (DHA) & $2 \cdot 00$ & 1.02 & $7 \cdot 86^{\star}$ & 3.78 & 2.40 & 0.40 & $5 \cdot 54^{\star}$ & 2.74 \\
\hline Sum of $n-3$ PUFA & $2 \cdot 17$ & 0.21 & $9 \cdot 70^{*}$ & 0.79 & $2 \cdot 60$ & 0.07 & $6 \cdot 64^{*}$ & 0.57 \\
\hline
\end{tabular}

LA, linoleic acid; GLA, $\gamma$-linolenic acid; DHGLA, dihomo- $\gamma$-linolenic acid; AA, arachidonic acid; ALA, $\alpha$-linolenic acid; DPA, docosapentaenoic acid.

* Mean value is significantly different from that of the CTRL animals $(P<0.05)$.

t Mean value is significantly different from that of the PBS-treated animals $(P<0.05)$

$\ddagger$ Data are percentages $(\mathrm{w} / \mathrm{w})$ calculated on the basis of six independent samples.

n-6 PUFA. Values of linoleic acid increased significantly in cholesteryl esters, but decreased in TAG. Similarly, $\gamma$-linolenic acid values were higher in phospholipids, but significantly lower in TAG and cholesteryl esters in the animals treated with $n$-3 PUFA than in the controls. Values of DHGLA were significantly higher in both cholesteryl esters and phospholipids in animals treated with $n-3$ PUFA than in control mice. In contrast, percentages (w/w) of AA were significantly lower in all three serum lipid classes of animals receiving $n$-3 PUFA supplementation than in the controls. The sum of $n-6$ PUFA was always lower in animals receiving $n$-3 PUFA supplementation.

Table 4. Fatty acid composition of plasma triacylglycerol lipids in mice fed either with a diet supplemented with docosahexaenoic acid-enriched fish oil or a control diet (CTRL) and subsequently treated either with ovalbumin (OVA) or phosphate-buffered salineł

(Mean values and standard deviations)

\begin{tabular}{|c|c|c|c|c|c|c|c|c|}
\hline \multirow{2}{*}{$\begin{array}{l}\text { Treatment... } \\
\text { Fatty acid }\end{array}$} & \multicolumn{2}{|c|}{ CTRL-PBS } & \multicolumn{2}{|c|}{ DHA-PBS } & \multicolumn{2}{|c|}{ CTRL-OVA } & \multicolumn{2}{|c|}{ DHA-OVA } \\
\hline & Mean & SD & Mean & SD & Mean & SD & Mean & $\mathrm{SD}$ \\
\hline Sum of SFA & $25 \cdot 21$ & 0.30 & $26 \cdot 91$ & 0.70 & $23 \cdot 18$ & 0.34 & $34 \cdot 12^{\star} \dagger$ & 0.83 \\
\hline Sum of MUFA & $36 \cdot 24$ & 0.46 & $30 \cdot 39^{*}$ & 0.51 & $40 \cdot 39$ & 0.52 & $29 \cdot 70^{\star}$ & 0.56 \\
\hline $18: 2 n-6$ (LA) & $33 \cdot 20$ & $2 \cdot 58$ & $27 \cdot 19$ & 4.47 & 31.51 & 4.42 & $22 \cdot 06^{\star}$ & $2 \cdot 34$ \\
\hline $18: 3 n-6$ (GLA) & 0.27 & 0.05 & $0.16^{*}$ & 0.04 & 0.27 & 0.03 & 0.27 & 0.20 \\
\hline $20: 2 n-6$ & 0.53 & 0.20 & $0 \cdot 26^{*}$ & 0.04 & 0.47 & 0.12 & $0.25^{\star}$ & 0.03 \\
\hline $20: 3 n-6$ (DHGLA) & 0.64 & 0.29 & 0.40 & 0.17 & 0.49 & 0.11 & 0.36 & 0.09 \\
\hline $20: 4 n-6(\mathrm{AA})$ & 2.53 & 0.91 & $1.02^{*}$ & 0.26 & $2 \cdot 44$ & 0.31 & $0.99^{\star}$ & 0.64 \\
\hline $22: 4 n-6$ & 0.54 & 0.21 & 0.49 & 0.20 & 0.47 & 0.13 & 0.43 & 0.12 \\
\hline $22: 5 n-6$ & 0.41 & 0.26 & 0.39 & 0.30 & 0.19 & 0.19 & $2 \cdot 19^{\star}$ & 1.84 \\
\hline Sum of $n-6$ PUFA & $38 \cdot 11$ & 0.49 & $29 \cdot 91^{*}$ & $1 \cdot 14$ & $35 \cdot 84$ & $1 \cdot 11$ & $26 \cdot 54^{\star}$ & 0.59 \\
\hline $18: 3 n-3(\mathrm{ALA})$ & 0.04 & 0.03 & $0 \cdot 19^{\star}$ & 0.02 & $0.11 \dagger$ & 0.05 & $0.24^{\star}$ & 0.04 \\
\hline $20: 3 n-3$ & 0.26 & 0.19 & 0.32 & 0.17 & 0.26 & 0.27 & 0.57 & 0.34 \\
\hline $20: 5 n-3$ (EPA) & 0.02 & 0.01 & $1.47^{*}$ & 0.42 & $0.01 \dagger$ & 0.01 & $1 \cdot 20^{*}$ & 0.55 \\
\hline $22: 5 n-3$ (DPA) & 0.09 & 0.03 & $0.87^{*}$ & 0.45 & $0.03 \dagger$ & 0.01 & $0.72^{*}$ & 0.25 \\
\hline $22: 6 n-3(\mathrm{DHA})$ & 0.03 & 0.01 & $9 \cdot 94^{*}$ & $2 \cdot 64$ & $0.18 \dagger$ & 0.02 & $6 \cdot 92^{*}$ & $3 \cdot 64$ \\
\hline Sum of $n-3$ PUFA & 0.43 & 0.05 & $12 \cdot 80^{\star}$ & 0.62 & 0.59 & 0.07 & $9 \cdot 64^{*}$ & 0.83 \\
\hline
\end{tabular}

LA, linoleic acid; GLA, $\gamma$-linolenic acid; DHGLA, dihomo- $\gamma$-linolenic acid; AA, arachidonic acid; ALA, $\alpha$-linolenic acid; DPA, docosapentaenoic acid.

${ }^{*}$ Mean value is significantly different from that of the CTRL animals $(P<0.05)$.

† Mean value is significantly different from that of the PBS-treated animals $(P<0.05)$.

Data are percentages $(\mathrm{w} / \mathrm{w})$ calculated on the basis of six independent samples. 
Table 5. Ratios of selected $n-3$ and $n-6$ polyunsaturated fatty acids $\ddagger$ (Mean values and standard deviations)

\begin{tabular}{|c|c|c|c|c|c|c|}
\hline & \multicolumn{2}{|c|}{ EPA:AA } & \multicolumn{2}{|c|}{ EPA:DHGLA } & \multicolumn{2}{|c|}{ EPA:DHA } \\
\hline & Mean & SD & Mean & SD & Mean & SD \\
\hline \multicolumn{7}{|l|}{ TAG } \\
\hline CTRL-PBS (\%)§ & 100 & 41 & 100 & 73 & 100 & 42 \\
\hline CTRL-OVA (\%) & $23^{\star}$ & 26 & $20^{*}$ & 19 & $3^{*}$ & 4 \\
\hline DHA-PBS (\%)§ & 100 & 26 & 100 & 37 & 100 & 19 \\
\hline DHA-OVA (\%) & 70 & 31 & $92 \dagger$ & 51 & 119† & 11 \\
\hline \multicolumn{7}{|l|}{ Cholesteryl esters } \\
\hline CTRL-PBS (\%)§ & 100 & 106 & 100 & 91 & 100 & 105 \\
\hline CTRL-OVA (\%) & 29 & 6 & 42 & 15 & 26 & 6 \\
\hline DHA-PBS (\%)§ & 100 & 21 & 100 & 34 & 100 & 16 \\
\hline DHA-OVA (\%) & $80 \dagger$ & 18 & 66 & 26 & $88 †$ & 17 \\
\hline \multicolumn{7}{|l|}{ Phospholipids } \\
\hline CTRL-PBS (\%)§ & 100 & 92 & 100 & 79 & 100 & 122 \\
\hline CTRL-OVA (\%) & 29 & 15 & 36 & 22 & 19 & 9 \\
\hline DHA-PBS (\%)§ & 100 & 21 & 100 & 23 & 100 & 46 \\
\hline DHA-OVA (\%) & $76 \dagger$ & 17 & $68^{*} \dagger$ & 13 & 93† & 26 \\
\hline
\end{tabular}

AA, arachidonic acid; DHGLA, dihomo- $\gamma$-linolenic acid; CTRL, control diet; OVA, treatment with ovalbumin.

* Mean value is significantly different from that of the PBS-treated animals $(P<0.05)$.

† Mean value is significantly different from that of the CTRL animals $(P<0.05)$.

$\ddagger$ Data are calculated on the basis of six independent samples.

$\S$ Ratios detected in PBS-treated groups were set as $100 \%$.

Effect of dietary docosahexaenoic acid-enriched fish oil supplementation with allergic sensitisation (control diet and ovalbumin treatment $\mathrm{v}$. docosahexaenoic acid-enriched diet and ovalbumin treatment)

Values of the relevant $n$-3 PUFA were all significantly higher in the animals supplemented with DHA and EPA. The sums of SFA were significantly higher in all serum lipid classes in the animals receiving $n-3$ PUFA supplementation. The sums of MUFA were higher in phospholipids and cholesteryl esters, but were significantly lower in TAG in the animals treated with $n-3$ PUFA than in the controls. Values of linoleic acid were significantly higher in cholesteryl esters, but significantly lower in TAG in the animals receiving DHA and EPA supplementation. AA values as well as the sum of $n-6$ PUFA were significantly lower in all serum lipid classes in the animals treated with $n-3$ PUFA than in the controls. In general, all $n$-3 PUFA levels were higher after DHA supplementation.

\section{Effect of allergic sensitisation without dietary docosahexaenoic acid-enriched fish oil supplementation (control diet and phosphate-buffered saline treatment $\mathrm{v}$. control diet and ovalbumin treatment)}

The sums of SFA were comparable in phospholipids and TAG in sensitised and non-sensitised animals, while in cholesteryl esters they were significantly lower in the sensitised animals. The sums of MUFA were similar in phospholipids, TAG and cholesteryl esters. Allergic sensitisation did not result in significant changes in $n$ - 6 PUFA values. $n$ - 3 PUFA values exhibited significant changes upon sensitisation mainly in TAG: values of $\alpha$-linolenic acid and DHA significantly increased, whereas those of EPA and docosapentaenoic acid (22: 5n-3) significantly decreased. EPA was significantly lower in TAG and phospholipids and borderline significantly lower in cholesteryl esters.

Effect of allergic sensitisation with dietary docosahexaenoic acid-enriched fish oil supplementation (docosahexaenoic acidenriched diet and phosphate-buffered saline treatment $\mathrm{v}$. docosahexaenoic acid-enriched diet and ovalbumin treatment)

Sensitisation with OVA with dietary interventions resulted in similar SFA levels in cholesteryl esters but significantly increased levels in TAG and phospholipids. The sums of MUFA were similar in all lipid classes. $n-6$ PUFA values were similar in cholesteryl esters and TAG, while in phospholipids the sum of $n-6$ PUFA as well as linoleic acid levels were significantly lower in the sensitised animals.

\section{Inter-comparison of lipid classes}

DHA supplementation affected the fatty acid compositions of all three lipid classes $v$. control. The increase in the sum of $n-3$ PUFA expressed as DHA-PBS/CTRL-PBS was the most pronounced in serum TAG (thirty times), in comparison with phospholipids (four times) and cholesteryl esters (six times).

Table 6. Fatty acid composition of plasma phospholipids in mice fed either with a diet supplemented with docosahexaenoic acid-enriched fish oil or a control diet (CTRL) and subsequently treated either with ovalbumin (OVA) or phosphate-buffered saline and ratios of selected $n-3$ and $n-6$ polyunsaturated fatty acids $\ddagger$

(Mean values and standard deviations)

\begin{tabular}{|c|c|c|c|c|c|c|c|c|c|c|c|c|c|c|}
\hline \multirow[t]{2}{*}{ Fatty acid... } & \multicolumn{2}{|c|}{$\mathrm{AA}$} & \multicolumn{2}{|c|}{ DHGLA } & \multicolumn{2}{|c|}{$\mathrm{DHA}$} & \multicolumn{2}{|c|}{ EPA } & \multicolumn{2}{|c|}{$\begin{array}{c}\text { EPA:AA } \\
(\%) \S\end{array}$} & \multicolumn{2}{|c|}{$\begin{array}{c}\text { EPA:DHGLA } \\
(\%) \S\end{array}$} & \multicolumn{2}{|c|}{$\begin{array}{c}\text { EPA:DHA } \\
(\%) \S\end{array}$} \\
\hline & Mean & SD & Mean & SD & Mean & SD & Mean & SD & Mean & SD & Mean & SD & Mean & SD \\
\hline CTRL-PBS & $14 \cdot 0$ & 3.6 & 1.00 & 0.19 & $2 \cdot 00$ & 1.02 & 0.007 & 0.005 & 100 & 92 & 100 & 79 & 100 & 122 \\
\hline $\mathrm{CTRL}-\mathrm{Al}(\mathrm{OH})_{3}$ & $11 \cdot 2$ & 0.8 & 0.88 & 0.08 & $2 \cdot 08$ & 0.30 & 0.010 & 0.006 & 128 & 59 & 155 & 83 & 82 & 44 \\
\hline CTRL-OVA-AI $(\mathrm{OH})_{3}$ & $15 \cdot 9$ & $2 \cdot 0$ & 1.03 & 0.12 & $2 \cdot 40$ & 0.40 & 0.003 & 0.001 & 29 & 15 & 36 & 22 & 19 & 9 \\
\hline DHA-PBS & $6 \cdot 10^{*}$ & $1 \cdot 78$ & $1.63^{*}$ & 0.30 & $7 \cdot 86^{*}$ & $3 \cdot 78$ & $0.94^{*}$ & 0.32 & 100 & 21 & 100 & 23 & 100 & 46 \\
\hline DHA-OVA-Al(OH) $)_{3}$ & $5 \cdot 48^{*}$ & 1.46 & $1.64^{*}$ & 0.32 & $5 \cdot 54^{*}$ & $2 \cdot 74$ & $0.64^{*}$ & 0.17 & $76^{*}$ & 17 & $68^{*} \dagger$ & 13 & $93^{\star}$ & 26 \\
\hline
\end{tabular}

AA, arachidonic acid; DHGLA, dihomo- $\gamma$-linolenic acid; OVA, treatment with ovalbumin

* Mean value is significantly different from that of the PBS-treated animals $(P<0.05)$.

† Mean value is significantly different from that of the CTRL animals $(P<0.05)$.

$\ddagger$ Data are percentages $(\mathrm{w} / \mathrm{w})$ calculated on the basis of six independent samples.

$\S$ Ratios detected in PBS-treated groups were set as $100 \%$. 
Eicosapentaenoic acid:arachidonic acid, eicosapentaenoic acid:dihomo- $\gamma$-linolenic acid and eicosapentaenoic acid:docosahexaenoic acid ratios

The ratios in the PBS-treated animals were always set as $100 \%$ (Table 5) for better comparison of the OVA sensitisation values after the basal diet or the diet supplemented with DHA-enriched fish oil. The EPA:AA ratio for TAG decreased significantly and just non-significantly in cholesteryl esters and phospholipids in non-supplemented animals to 23 (SD 26), 29 (SD 6) and 29 (SD 15) \% after allergic sensitisation, whereas the decrease was only to 70 (SD 31), 80 (SD 18) and 76 (SD 17) \% after accompanying DHAenriched fish oil supplementation.

The EPA:DHGLA ratio also decreased in non-supplemented animals to 20 (SD 18) \% for TAG (significantly), 42 (SD 15) \% for cholesteryl esters and 36 (SD 22) \% for phospholipids after allergic sensitisation, whereas the decrease was much smaller (to 92 (SD 51) \% for TAG, 66 (SD 26) \% for cholesteryl esters and 68 (SD 13) \% for phospholipids) after DHA-enriched fish oil supplementation.

The EPA:DHA ratio decreased after sensitisation without DHA-enriched fish oil supplementation to 3 (SD 4) \% significantly for TAG, 26 (SD 6) \% for cholesteryl esters and 19 (SD 9) $\%$ for phospholipids of the original value, while after DHA-enriched fish oil supplementation it was significantly higher after sensitisation with 119 (SD 11) \% for TAG, 88 (SD 17) $\%$ for cholesteryl esters and 93 (SD 26) \% for phospholipids.

Influence of aluminium hydroxide treatment on the fatty acid composition of plasma phospholipids

Concentrations of AA, DHGLA, DHA and EPA were not significantly altered after $\mathrm{Al}(\mathrm{OH})_{3}$ or $\mathrm{Al}(\mathrm{OH})_{3}$-OVA treatments in the animals fed the basal diet as well as in the $n$-3 PUFA-supplemented animals. Concentrations of EPA were always lower (non-significantly) in $\mathrm{OVA}-\mathrm{Al}(\mathrm{OH})_{3}$-treated mice in comparison with PBS-treated mice.

EPA:AA, EPA:DHGLA and EPA:DHA ratios were in the same range after $\mathrm{Al}(\mathrm{OH})_{3}$ treatment in animals fed the basal diet as for animals with PBS treatment, while the ratios were much, but non-significantly, lower in $\mathrm{OVA}-\mathrm{Al}(\mathrm{OH})_{3}$-treated animals. EPA:AA, EPA:DHGLA and EPA:DHA ratios were just slightly lower after $\mathrm{Al}(\mathrm{OH})_{3}$ treatment; EPA:AA and EPA:DHA ratios were lower and just EPA:DHGLA ratios were significantly lower in the $n$-3 PUFA diet-supplemented mice.

The $n$-3 PUFA-supplemented diet significantly ameliorated the decrease of the EPA:AA, EPA:DHGLA and EPA:DHA ratios in plasma phospholipids after $\mathrm{OVA}-\mathrm{Al}(\mathrm{OH})_{3}$ treatment but not after $\mathrm{Al}(\mathrm{OH})_{3}$ treatment alone in comparison with animals fed the basal diet.

\section{Allergic sensitisation}

Serum IgE levels increased significantly after OVA sensitisation in the group fed the control diet from 0.4 (SD 0.2$) \mu \mathrm{g} / \mathrm{ml}$ to 3.2 (SD 2.0) $\mu \mathrm{g} / \mathrm{ml}$ and in the DHA-enriched fish oil-supplemented group from 0.7 (SD 0.1$) \mu \mathrm{g} / \mathrm{ml}$ to 2.8 (SD 1.3 ) $\mu \mathrm{g} / \mathrm{ml}$. There was no significant difference between the IgE levels of the group fed the control diet and the DHA-enriched fish oil-supplemented group. Additionally the specificity of the sensitisation was supported by measuring OVA-specific IgE titres in sensitised mice whereas in non-sensitised mice no OVA-specific IgE was detected (data not shown; C Koch, S Dölle, M Metzger, C Rasche, H Jurydas, R Rühl, H Renz and M Worm, unpublished results).

\section{Discussion}

In the present study, we demonstrated that both DHA-enriched fish oil supplementation and allergic sensitisation significantly influence the fatty acid composition of different serum lipid classes. Allergic sensitisation after three intraperitoneal injections of OVA associated to $\mathrm{Al}(\mathrm{OH})_{3}$ has been shown in various publications of our group ${ }^{(27,29,30)}$ and additionally in the present study we determined an increase of total IgE after OVA sensitisation. The levels of IgE were just slightly lower in the DHAenriched fish oil-supplemented group in comparison with the control diet-fed group. A following publication will describe more deeply the effects of DHA on immuno-relevant parameters (C Koch, S Dölle, R Rühl and M Worm, unpublished results). In the present paper we first describe the alteration of ratios of individual fatty acids being precursors of pro-inflammatory or anti-inflammatory PUFA metabolites changed after allergic sensitisation in a manner depending on DHA-enriched fish oil supplementation.

Supplementation of DHA has been shown in various studies carried out in various organisms to yield in increased concentrations of DHA as well as EPA in serum lipids ${ }^{(17,18,31)}$. Our data support this aspect of previous reports in a mouse model investigating the fatty acid composition of serum TAG, phospholipids and cholesteryl esters. The effect of DHA-enriched fish oil supplementation on EPA levels could be mediated via three pathways: (1) increased concentrations of EPA in the DHA-enriched fish oil; (2) retroconversion of DHA to $\mathrm{EPA}^{(7)} ;(3)$ decreased conversion of EPA to $\mathrm{DHA}^{(7)}$. In addition to increased levels of the principal $n$-3 long-chain PUFA DHA and EPA, the levels of the $n-6$ long-chain PUFA AA markedly decreased. This finding is in accordance with previous observations $^{(17,31)}$, and may reflect the inhibitory effects of abundance of DHA on $\Delta$-6-desaturase, the rate-limiting step also in AA biosynthesis (for a review, see Horrobin ${ }^{(32)}$ ), based on higher concentrations of AA precursors in sunflower-seed oil in comparison with DHA-enriched fish oil (Table 1).

The exact interrelationship of fatty acids in circulating serum lipids to tissue fatty acid metabolism remains to be clarified $^{(33,34)}$. However, fatty acid composition of serum lipid classes is still considered an important indicator of fatty acid status ${ }^{(35)}$. In the present study, effects of dietary intervention were detectable in all the three serum lipid classes analysed, whereas a marked effect of allergic sensitisation on $n$-3 long-chain PUFA was seen mainly in serum TAG esters.

The major outcome of the present study was the influence of the allergic sensitisation on the fatty acid composition of various serum lipid classes. To the best of our knowledge, no previous study has focused on the effect of allergic sensitisation on serum fatty acid patterns, whereas several studies have compared fatty acid status in human subjects with and without allergic disease $^{(13,36)}$ (for recent reviews, see Devereux \& Seaton ${ }^{(11)}$ and Mickleborough \& Rundell $\left.{ }^{(12)}\right)$. Possibly these alterations are partly due to an altered lipoprotein distribution after the acute-phase response (for a review, see Schweigert ${ }^{(37)}$ ). 
In the present study, we investigated the effects of allergic sensitisation induced by triple intraperitoneal OVA injections in adult mice, and observed several significant alterations of plasma phospholipids fatty acid levels. Our experiments showed that $\mathrm{Al}(\mathrm{OH})_{3}$ has no or just marginal effects on lipid composition. This biochemical inactive $\mathrm{Al}(\mathrm{OH})_{3}$ adjuvant is used to potentiate the immune responses to vaccines by adsorbing the antigen ${ }^{(38,39)} \cdot \mathrm{Al}(\mathrm{OH})_{3}$ does not alter serum lipid concentrations if given intraperitoneally and absorbed to allergen, while $\mathrm{Al}$ or $\mathrm{Al}$ ions could be quite active in the alteration of lipid metabolism ${ }^{(40)}$. No relevant amounts of $\mathrm{Al}$ and $\mathrm{Al}$ ions will be reached in the blood circulation when biochemically stable $\mathrm{Al}(\mathrm{OH})_{3}$ is used under physiological $\mathrm{pH}$ conditions after intraperitoneal injections ${ }^{(41)}$.

After allergic sensitisation but without accompanying DHA supplementation, the levels of mainly the $n$-3 PUFA, $\alpha$-linolenic acid, EPA, docosapentaenoic acid and DHA were significantly altered in TAG. EPA and docosapentaenoic acid were significantly down regulated, whereas DHA and $\alpha$-linolenic acid were significantly up regulated. For better visualisation of the results obtained, the ratios of precursors of pro- and anti-inflammatory bioactive lipids EPA, DHGLA and AA were calculated in Table 5.

EPA:AA and EPA:DHGLA ratios were significantly reduced upon allergic sensitisation and without accompanying DHAenriched fish oil supplementation. After accompanying DHAenriched fish oil supplementation, however, the reduction of the ratios was much lower. Consequently, the ratios were significantly lower in the non-supplemented than in supplemented animals.

Metabolism via COX and LOX pathways has been shown to be highly dependent on the availability of lipid precursors for further metabolism ${ }^{(42)}$. It has been convincingly demonstrated that $n$-3 PUFA were better substrates for the conversion by $\mathrm{LOX}^{(43)}$, but much weaker for the COX-2-mediated pathways to $\mathrm{PgE}$ derivatives ${ }^{(44)}$. In addition, leucotrienes originating from EPA have been shown to be much less active in comparison with analogues from the AA cascade ${ }^{(45)}$. EPA and other $n$-3 PUFA were also found to inhibit COX and LOX activity ${ }^{(46-48)}$.

Our data strongly support the hypothesis that DHA-enriched fish oil supplementation significantly alters the levels and ratios of $n-3: n-6$ precursor fatty acids for further bioactivation to pro-inflammatory $\mathrm{PG}$, which are mainly $\mathrm{T}$-helper cell 2 skewing, and leucotrienes ${ }^{(4,49)}$. Various immune-competent cells such as lymphocytes, macrophages, dendritic cells, etc could alter lipid metabolism via various enzymes such as LOX, COX, cytochromes, etc, as well as various immune reactions could be influenced by dietary lipids and their active metabolites (summarised in various reviews ${ }^{(3,50)}$ ). Further animal studies are in progress as well as the establishment of sensitive MS-based analytical techniques to identify bioactive eicosanoids in in vitro as well as in vivo studies in the serum and various immunocompetent cells at various stages during allergic sensitisation. In addition, novel studies by Serhan's group support that EPA and DHA via COXand LOX-driven pathways serve as precursors for anti-inflammatory bioactive lipids such as lipoxins, neuroprotectins and resolvins (for reviews, see Serhan ${ }^{(5)}$ and Serhan \& Savill ${ }^{(6)}$ ). Higher levels of DHA and EPA may lead to increased production of these novel-described derivatives in the mammalian organism after DHA-enriched fish oil supplementation and accompanying allergic sensitisation, possibly resulting in reduced severity of the allergic phenotype.

In summary, the data obtained in the present study indicate that fatty acid levels and especially the ratios of fatty acids representing precursors of bioactive lipids after allergic sensitisation highly depend on accompanying DHA-enriched fish oil supplementation. This DHA-enriched fish oil supplementation-mediated alteration of lipid ratios of bioactive-precursor lipids may explain the allergy-ameliorating effects of DHA in particular and/or $n-3$ PUFA in general ${ }^{(14-20)}$; unfortunately in our experimental set up we could not observe any allergyameliorating effect. Further studies of our group will focus on lipid metabolism and molecular mechanisms of possible allergy-preventive effects of DHA in animal studies, human supplementation trials and human cohort studies.

\section{Acknowledgements}

R. R. and J. M. have been financially supported by funds from the EU-RTN 'Nutriceptors' programme. R. R., F. J. S. and M. W. directed the research, R. R. analysed the data and wrote the paper, C. K. and J. M. performed the animal experimental work and T. M. performed the analytical work. The authors declare no conflict of interest.

\section{References}

1. Jump DB (2002) The biochemistry of $n-3$ polyunsaturated fatty acids. J Biol Chem 277, 8755-8758.

2. Siddiqui RA, Shaikh SR, Sech LA, Yount HR, Stillwell W \& Zaloga GP (2004) Omega 3-fatty acids: health benefits and cellular mechanisms of action. Mini Rev Med Chem 4, 859-871.

3. Rustan AC \& Drevon CA (2001) Fatty acids: structures and properties. In Encyclopedia of Life Sciences. Chichester: John Wiley \& Sons.

4. Miles EA, Aston L \& Calder PC (2003) In vitro effects of eicosanoids derived from different 20-carbon fatty acids on $\mathrm{T}$ helper type 1 and T helper type 2 cytokine production in human wholeblood cultures. Clin Exp Allergy 33, 624-632.

5. Serhan CN (2005) Novel omega-3-derived local mediators in anti-inflammation and resolution. Pharmacol Ther 105, 7-21.

6. Serhan CN \& Savill J (2005) Resolution of inflammation: the beginning programs the end. Nat Immunol 6, 1191-1197.

7. Hansen JB, Grimsgaard S, Nilsen H, Nordoy A \& Bonaa KH (1998) Effects of highly purified eicosapentaenoic acid and docosahexaenoic acid on fatty acid absorption, incorporation into serum phospholipids and postprandial triglyceridemia. Lipids 33, $131-138$.

8. Stulnig TM (2003) Immunomodulation by polyunsaturated fatty acids: mechanisms and effects. Int Arch Allergy Immunol 132, 310-321.

9. James MJ, Gibson RA \& Cleland LG (2000) Dietary polyunsaturated fatty acids and inflammatory mediator production. Am J Clin Nutr 71, Suppl. 1, 343S-348S.

10. Mishra A, Chaudhary A \& Sethi S (2004) Oxidized omega-3 fatty acids inhibit NF- $\mathrm{B}$ activation via a PPAR $\alpha$-dependent pathway. Arterioscler Thromb Vasc Biol 24, 1621-1627.

11. Devereux G \& Seaton A (2005) Diet as a risk factor for atopy and asthma. J Allergy Clin Immunol 115, 1109-1118.

12. Mickleborough TD \& Rundell KW (2005) Dietary polyunsaturated fatty acids in asthma- and exercise-induced bronchoconstriction. Eur J Clin Nutr 59, 1335-1346.

13. Hoff S, Seiler H, Heinrich J, et al. (2005) Allergic sensitisation and allergic rhinitis are associated with $n-3$ polyunsaturated 
fatty acids in the diet and in red blood cell membranes. Eur $J$ Clin Nutr 59, 1071-1080.

14. Nagakura T, Matsuda S, Shichijyo K, Sugimoto H \& Hata K (2000) Dietary supplementation with fish oil rich in omega-3 polyunsaturated fatty acids in children with bronchial asthma. Eur Respir J 16, 861-865.

15. Watanabe T \& Kuroda Y (1999) The effect of a newly developed ointment containing eicosapentaenoic acid and docosahexaenoic acid in the treatment of atopic dermatitis. J Med Invest 46, 173-177

16. Abba C, Mussa PP, Vercelli A \& Raviri G (2005) Essential fatty acids supplementation in different-stage atopic dogs fed on a controlled diet. J Anim Physiol Anim Nutr (Berl) 89, 203-207.

17. Mueller RS, Fettman MJ, Richardson K, Hansen RA, Miller A, Magowitz J \& Ogilvie GK (2005) Plasma and skin concentrations of polyunsaturated fatty acids before and after supplementation with $n-3$ fatty acids in dogs with atopic dermatitis. Am J Vet Res 66, $868-873$.

18. Mueller RS, Fieseler KV, Fettman MJ, Zabel S, Rosychuk RA, Ogilvie GK \& Greenwalt TL (2004) Effect of omega-3 fatty acids on canine atopic dermatitis. J Small Anim Pract 45, 293-297.

19. Zhang P, Smith R, Chapkin RS \& McMurray DN (2005) Dietary $(n-3)$ polyunsaturated fatty acids modulate murine Th1/Th2 balance toward the Th2 pole by suppression of Th1 development. J Nutr 135, 1745-1751.

20. Yokoyama A, Hamazaki T, Ohshita A, Kohno N, Sakai K, Zhao GD, Katayama H \& Hiwada K (2000) Effect of aerosolized docosahexaenoic acid in a mouse model of atopic asthma. Int Arch Allergy Immunol 123, 327-332.

21. Worm M \& Henz BM (1997) Molecular regulation of human IgE synthesis. $J$ Mol Med 75, 440-447.

22. Bacharier LB \& Geha RS (2000) Molecular mechanisms of IgE regulation. J Allergy Clin Immunol 105, S547-S558.

23. Fogh K, Herlin T \& Kragballe K (1989) Eicosanoids in skin of patients with atopic dermatitis: prostaglandin $\mathrm{E} 2$ and leukotriene B4 are present in biologically active concentrations. J Allergy Clin Immunol 83, 450-455.

24. Koro O, Furutani K, Hide M, Yamada S \& Yamamoto S (1999) Chemical mediators in atopic dermatitis: involvement of leukotriene B4 released by a type I allergic reaction in the pathogenesis of atopic dermatitis. J Allergy Clin Immunol 103, 663-670.

25. Lee TH, Menica-Huerta JM, Shih C, Corey EJ, Lewis RA \& Austen KF (1984) Characterization and biologic properties of 5,12-dihydroxy derivatives of eicosapentaenoic acid, including leukotriene B5 and the double lipoxygenase product. $J$ Biol Chem 259, 2383-2389.

26. Schweigert FJ, Trupschuch A \& Hantschel C (2002) Modulation of absorption of $\beta$-carotene and tissue accumulation of $\beta$-carotene and vitamin A by different surfactants in rats. Ann Nutr Metab 46, 200-204.

27. Rühl R, Dahten A, Schweigert FJ, Herz U \& Worm M (2003) Inhibition of IgE-production by peroxisome proliferator-activated receptor ligands. J Invest Dermatol 121, 757-764.

28. Folch J, Lees M \& Sloane Stanley GH (1957) A simple method for the isolation and purification of total lipides from animal tissues. J Biol Chem 226, 497-509.

29. Rühl R, Garcia A, Schweigert FJ \& Worm M (2004) Modulation of cytokine production by low and high retinoid diets in ovalbumin-sensitized mice. Int J Vitam Nutr Res 74, 279-284.

30. Worm M, Herz U, Krah JM, Renz H \& Henz BM (2001) Effects of retinoids on in vitro and in vivo IgE production. Int Arch Allergy Immunol 124, 233-236.

31. Kew S, Mesa MD, Tricon S, Buckley R, Minihane AM \& Yaqoob P (2004) Effects of oils rich in eicosapentaenoic and docosahexaenoic acids on immune cell composition and function in healthy humans. Am J Clin Nutr 79, 674-681.
32. Horrobin DF (1993) Fatty acid metabolism in health and disease: the role of $\Delta$-6-desaturase. Am J Clin Nutr 57, Suppl. 5, 732S-737S.

33. Di Stasi D, Bernasconi R, Marchioli R, Marfisi RM, Rossi G, Tognoni G \& Tacconi MC (2004) Early modifications of fatty acid composition in plasma phospholipids, platelets and mononucleates of healthy volunteers after low doses of $n-3$ polyunsaturated fatty acids. Eur J Clin Pharmacol 60, 183-190.

34. Nikolaidis MG, Petridou A \& Mougios V (2005) Comparison of the phospholipid and triacylglycerol fatty acid profile of rat serum, skeletal muscle and heart. Physiol Res 55, 259-265.

35. Aro A (2003) Fatty acid composition of serum lipids: is this marker of fat intake still relevant for identifying metabolic and cardiovascular disorders? Nutr Metab Cardiovasc Dis 13, 253-255.

36. Korotkova M, Telemo E, Yamashiro Y, Hanson LA \& Strandvik B (2004) The ratio of $n-6$ to $n-3$ fatty acids in maternal diet influences the induction of neonatal immunological tolerance to ovalbumin. Clin Exp Immunol 137, 237-244.

37. Schweigert FJ (2001) Inflammation-induced changes in the nutritional biomarkers serum retinol and carotenoids. Curr Opin Clin Nutr Metab Care 4, 477-481.

38. Verdier F, Burnett R, Michelet-Habchi C, Moretto P, FievetGroyne F \& Sauzeat E (2005) Aluminium assay and evaluation of the local reaction at several time points after intramuscular administration of aluminium containing vaccines in the Cynomolgus monkey. Vaccine 23, 1359-1367.

39. Jefferson T, Rudin M \& Di Pietrantonj C (2004) Adverse events after immunisation with aluminium-containing DTP vaccines: systematic review of the evidence. Lancet Infect Dis 4, 84-90.

40. Sarin S, Gupta V \& Gill KD (1997) Alterations in lipid composition and neuronal injury in primates following chronic aluminium exposure. Biol Trace Elem Res 59, 133-143.

41. Berthon G \& Dayde S (1992) Why aluminum phosphate is less toxic than aluminum hydroxide. $J$ Am Coll Nutr 11, 340-348.

42. Caughey GE, Mantzioris E, Gibson RA, Cleland LG \& James MJ (1996) The effect on human tumor necrosis factor $\alpha$ and interleukin 1 (production of diets enriched in $n-3$ fatty acids from vegetable oil or fish oil. Am J Clin Nutr 63, 116-122.

43. Jakschik BA, Sams AR, Sprecher H \& Needleman P (1980) Fatty acid structural requirements for leukotriene biosynthesis. Prostaglandins 20, 401-410.

44. Malkowski MG, Thuresson ED, Lakkides KM, Rieke CJ, Micielli R, Smith WL \& Garavito RM (2001) Structure of eicosapentaenoic and linoleic acids in the cyclooxygenase site of prostaglandin endoperoxide H synthase-1. J Biol Chem 276, 37547-37555

45. Leitch AG, Lee TH, Ringel EW, Prickett JD, Robinson DR, Pyne SG, Corey EJ, Drazen JM, Austen KF \& Lewis RA (1984) Immunologically induced generation of tetraene and pentaene leukotrienes in the peritoneal cavities of menhadenfed rats. J Immunol 132, 2559-2565.

46. Saku N, Kobayashi J \& Kitamura S (1999) Eicosapentaenoic acid modulates arachidonic acid metabolism in rat alveolar macrophages activated by silica. Prostaglandins Leukot Essent Fatty Acids 61, 51-54.

47. Obata $\mathrm{T}$, Nagakura $\mathrm{T}$, Masaki $\mathrm{T}$, Maekawa $\mathrm{K}$ \& Yamashita $\mathrm{K}$ (1999) Eicosapentaenoic acid inhibits prostaglandin D2 generation by inhibiting cyclo-oxygenase- 2 in cultured human mast cells. Clin Exp Allergy 29, 1129-1135.

48. Corey EJ, Shih C \& Cashman JR (1983) Docosahexaenoic acid is a strong inhibitor of prostaglandin but not leukotriene biosynthesis. Proc Natl Acad Sci USA 80, 3581-3584.

49. Arcoleo F, Milano S, D'Agostino P \& Cillari E (1995) Effect of exogenous leukotriene B4 (LTB4) on BALB/c mice splenocyte production of Th1 and Th2 lymphokines. Int J Immunopharmacol 17, 457-463.

50. Calder PC (2001) Polyunsaturated fatty acids, inflammation, and immunity. Lipids 36, 1007-1024. 